\title{
Trabalho noturno, qualidade do sono e adoecimento de trabalhadores de enfermagem
}

\author{
Evening work, sleep quality and illness of nursing workers
}

Trabajo nocturno, calidad del sueño y enfermedades de trabajadores de enfermería

Ariane Naidon Cattani' ${ }^{\text {io }}$ https://orcid.org/0000-0002-3365-9237

Rosângela Marion da Silva ${ }^{1}$ io https:/orcid.org/0000-0003-3978-9654

Carmem Lúcia Colomé Beck' io https://orcid.orgy/0000-0001-9060-1923

Fernanda Moura D'Almeida Miranda² io https://orcid.org/0000-0001-7140-9557

Graziele de Lima Dalmolin' io https://orid.org/0000-0003-0985-5788

Silviamar Camponogara' ${ }^{1}$ is https://orcid.org/0000-0001-9342-3683

\section{Como citar:}

Cattani AN, Silva RM, Beck CL, Miranda FM, Dalmolin GL, Camponogara S. Trabalho noturno, qualidade do sono e adoecimento de trabalhadores de enfermagem. Acta Paul Enferm. 2021;34:eAPE00843.

DOI

http://dx.doi.org/10.37689/actaape/2021A000843

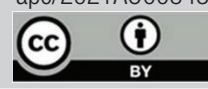

Descritores

Saúde do trabalhador; Jornada de trabalho em turnos; Sono; Riscos ocupacionais; Profissionais

de enfermagem

Keywords

Occupational health; Shift work schedule; Sleep; Occupational risks; Nurse practitioners

Descriptores

Salud laboral; Horario de trabajo por turnos; Sueño; Riesgos laborales; Enfermeras practicantes

Submetido 16 de Abril de 2020

Aceito 20 de Agosto de 2020

\section{Autor correspondente}

Ariane Naidon Cattan

E-mail: arianecattani@yahoo.com.br

\section{Resumo}

Objetivo: Analisar fatores associados à qualidade do sono e adoecimento em trabalhadores de enfermagem que atuam no turno noturno.

Métodos: Estudo transversal e correlacional realizado em hospital de ensino com uma amostra representativa de trabalhadores de enfermagem com atuação no noturno. Para coleta de dados utilizou-se um questionário sociolaboral, a Escala de Avaliação de Danos Relacionados ao Trabalho e o Índice de Qualidade do Sono de Pittsburgh. Utilizaram-se, para análise dos dados, teste Qui-Quadrado e correlação de Spearman, com níveis de significância de $5 \%$.

Resultados: Participaram 139 trabalhadores, com predomínio de adoecimento físico e com qualidade do sono ruim. Identificou-se associação entre qualidade do sono e as variáveis sexo e danos físicos. Adoecimento físico associou-se ao sexo, prática de atividade física, acidente de trabalho e afastamento do trabalho. Houve diferença estatística entre adoecimento psicológico e as variáveis sexo, tratamento de saúde, uso de medicação e prática de atividade física. Qualidade do sono correlacionou-se a danos físicos e psicológicos.

Conclusão: Os fatores identificados são úteis para planejar ações em saúde a fim de promover a saúde dos trabalhadores de enfermagem que atuam no turno noturno.

\section{Abstract}

Objective: To analyze factors associated with sleep quality and illness in nursing workers who work in the night shift.

Methods: Cross-sectional and correlational study carried out in a teaching hospital with a representative sample of nursing workers who work at night. For data collection, we used a socio-occupational questionnaire, the Work-Related Damage Assessment Scale and the Pittsburgh Sleep Quality Index. For data analysis, we used the Chi-square test and Spearman correlation, with significance level of $5 \%$.

Results: A total of 139 workers participated in the study, with a predominance of physical illness and poor sleep quality. An association was identified between sleep quality, gender and physical damage variables. Physical illness was associated with gender, physical activity, work accidents and work leave. There was a statistical difference between psychological illness and the variables gender, health treatment, use of medication and physical activity. Sleep quality correlated with physical and psychological damage.

Conclusion: The identified factors are useful for planning health actions in order to promote the health of nursing workers who work the night shift. 


\section{Resumen}

Objetivo: Analizar los factores asociados a la calidad del sueño y enfermedades de trabajadores de enfermería que actúan en el turno nocturno.

Métodos: Estudio transversal y correlacional realizado en un hospital universitario con una muestra representativa de trabajadores de enfermería en turno nocturno. Para la recolección de datos, se utilizó un cuestionario sociolaboral, la escala de Evaluación de Daños Relacionados con el Trabajo y el Índice de Calidad del Sueño de Pittsburgh. Para el análisis de los datos, se utilizó prueba $\chi^{2}$ de Pearson y correlación de Spearman, con nivel de significación de 5 \%.

Resultados: Participaron 139 trabajadores, con predominio de enfermedades físicas y sueño de mala calidad. Se identificó una relación entre calidad del sueño y variables sexo y daños físicos. Las enfermedades físicas se relacionaron con el sexo, la práctica de actividad física, accidentes laborales y ausencia al trabajo. Se observaron diferencias estadísticas entre enfermedades psicológicas y las variables sexo, tratamiento de salud, uso de medicación y práctica de actividad física. La calidad del sueño se correlacionó con daños físicos y psicológicos.

Conclusión: Los factores identificados son útiles para planificar acciones en salud a fin de promover la salud de los trabajadores de enfermería del turno nocturno.

\section{Introdução}

Os trabalhadores de saúde, especialmente os da enfermagem, estão expostos a elementos relacionados ao processo de trabalho que contribuem para um ambiente laboral complexo. Esses elementos podem incluir a realização do trabalho em turnos, particularmente no turno noturno, considerado preditor de alterações endócrinas e metabólicas, como aumento do índice de massa corpórea, ${ }^{(1)}$ aumento do risco de doença coronariana, ${ }^{(2)}$ prevalência da síndrome de Burnout, má qualidade e modificaçóes na duração do sono, ${ }^{(3-5)}$ o que sugere o adoecimento dos trabalhadores.

O trabalho no turno noturno pode impossibilitar a reposição adequada das horas de sono perdidas, considerando que o sono é uma necessidade fundamental para o relaxamento do corpo e restauraçáo física. Assim, atuar nesse turno, ter tempo reduzido para recuperação do sono e apresentar qualidade do sono ruim podem ser aspectos que contribuem para repercussões negativas na saúde física, emocional e psicossocial dos trabalhadores de enfermagem. ${ }^{(6,7)}$

Estudos nacionais e internacionais identificaram relação entre o trabalho noturno e a qualidade do sono ruim de trabalhadores de enfermagem. ${ }^{(8-10)} \mathrm{A}$ qualidade do sono influencia no desempenho de diversos processos fisiológicos, como sistema imunológico, metabolismo corporal, equilíbrio hormonal, saúde mental e emocional, podendo implicar fadiga, desequilíbrio físico e mental ${ }^{(7)}$ e danos à saúde.

Os danos à saúde podem ser classificados em físicos, definido como dores no corpo e distúrbios biológicos; psicológicos, como sentimentos negativos em relação a si mesmo e a vida em geral; e so- ciais, como isolamento e dificuldade nas relaçôes familiares e sociais. ${ }^{(11)}$ Essas situaçóes podem aumentar o risco para adoecimento, acidentes de trabalho e absenteísmo, pois essas adversidades decorrentes da organização laboral impactam na relação saúde-doença, que é expressa no biopsicossocial. ${ }^{(12)}$

Considerando o exposto e frente ao avanço de pesquisas referentes à saúde do trabalhador, que apontam o crescente adoecimento relacionado à atividade laboral em trabalhadores de enfermagem, ${ }^{(13)}$ questiona-se: quais fatores estão associados a qualidade do sono e adoecimento em trabalhadores de enfermagem que atuam no turno noturno? O objetivo deste estudo foi analisar fatores associados à qualidade do sono e adoecimento em trabalhadores de enfermagem que atuam no turno noturno.

\section{Métodos}

Estudo quantitativo, transversal e correlacional. Foi realizado em um hospital público de ensino, geral, de nível terciário, que atende $100 \%$ pelo Sistema Único de Saúde. Localizada no estado do Rio Grande do Sul, a instituição oferece 403 leitos de internação e o processo de trabalho ocorre diuturnamente e no turno noturno (das $07 \mathrm{~h}$ às $13 \mathrm{~h}$; das $13 \mathrm{~h}$ às $19 \mathrm{~h}$ e das $19 \mathrm{~h}$ às $07 \mathrm{~h}$ ). Sobre o regime de trabalho, há trabalhadores regidos pela Empresa Brasileira de Serviços Hospitalares, com jornada regular de trabalho: oito h diárias e/ou jornada especial de trabalho: $12 \mathrm{~h}$ consecutivas por $36 \mathrm{~h}$ de descanso, perfazendo $36 \mathrm{~h}$ semanais; ${ }^{(14)}$ e trabalhadores regidos pelo Regime Jurídico Único, com jornada de trabalho flexibilizada, perfazendo $30 \mathrm{~h}$ semanais. 
A coleta de dados ocorreu individualmente e no local de trabalho, no período de setembro de 2017 a abril de 2018, sendo estabelecido um período de cinco dias para a devolução dos instrumentos preenchidos. Os locais foram: clínica médica, unidade tocoginecológica, nefrologia, unidade de internaçáo pediátrica, unidade de terapia intensiva adulta e pediátrica, pronto socorro adulto e pediátrico, centro cirúrgico, sala de recuperação anestésica, centro obstétrico e centro de tratamento a criança com câncer.

No período da coleta de dados, a população de enfermagem era de 960 trabalhadores (333 enfermeiros, 500 técnicos de enfermagem e 127 auxiliares de enfermagem), que atuavam nos turnos diurno e noturno. Estipulou-se como critérios de inclusão: ser enfermeiro, técnico de enfermagem ou auxiliar de enfermagem, atuar na assistência direta aos usuários. E como critérios de exclusão: estar em licença ou afastamento de qualquer natureza durante o período da coleta de dados.

Foi realizado cálculo amostral, do tipo estratificado, por categoria profissional (enfermeiro, técnico de enfermagem e auxiliar de enfermagem), utilizando-se nível de confiança de $95 \%$, erro amostral de 5\%. A aplicação desses parâmetros produziu tamanho amostral mínimo de 277 trabalhadores de enfermagem. Após, verificou-se a proporção de cada categoria profissional na população $(35 \%$ de enfermeiros, 52\% técnicos de enfermagem e 13\% auxiliares de enfermagem). Tendo em vista a rotatividade nos turnos de trabalho, essa variável não pode ser considerada para fins de cálculo amostral. Assim, para este estudo, optou-se por avaliar somente os dados referentes aos trabalhadores que, no período de coleta de dados, atuavam no turno noturno.

Utilizou-se como instrumento de coleta dos dados um questionário sociolaboral para caracterizar os participantes (idade, sexo, filhos, situação conjugal, cargo na instituição, turno de trabalho, outro emprego, tempo de trabalho na unidade, pós-graduação, treinamento para atuar no setor, envolvimento com acidente de trabalho, satisfação com o trabalho e com a remuneração, opção pelo turno de trabalho, tratamento de saúde, uso de medicação, afastamento do trabalho por mo- tivo de doença, prática de atividade física, uso do tempo livre para lazer).

A Escala de Avaliação dos Danos Relacionados ao Trabalho (EADRT), validada, foi utilizada para avaliar os danos provocados pelo trabalho. A interpretação dos resultados é feita com base nas médias gerais dos fatores (danos físicos, psicológicos e sociais), sendo classificados em quatro níveis: suportável (abaixo de 1,9); crítico (entre 2,0 e 3,0); grave (entre 3,1 e 4,0) e presença de doenças ocupacionais (acima de 4,1). A avaliação suportável é a mais positiva, produtora de prazer no trabalho; o estado crítico indica uma situação limite, apontando sofrimento no trabalho e sinalizando estado de alerta; o nível grave é considerado produtor de sofrimento e indicador para grande risco de adoecimento, enquanto o mais elevado representa presença de doenças ocupacionais. Em nível crítico já significa adoecimento, portanto, realizou-se o agrupamento dessa classificação em não adoecimento (suportável) e adoecimento (crítico, grave e presença de doenças ocupacionais). ${ }^{(11)}$

Para mensurar a qualidade do sono utilizou-se o Índice de Qualidade do Sono de Pittsburgh, versão validada em português do Brasil (PSQI-BR). Sua pontuação global pode variar de 0 a 21 pontos, sendo caracterizado como qualidade do sono boa ( $\leq$ 4 ), qualidade do sono ruim (5-10) e presença de distúrbio do sono $(\geq 11){ }^{(15)}$ Optou-se por categorizar a variável em qualidade do sono "boa" $(\leq 5)$ e "ruim" (> 5 pontos), conforme identificado previamente. ${ }^{(16)}$

Os dados foram duplamente digitados no programa Excel for Windows/7 (Microsoft Office 2007) e analisados estatisticamente com o auxílio do Predictive Analytics Software, da SPSS, versão 18.0. As variáveis categóricas estão descritas por meio da frequência absoluta $(\mathrm{N})$ e relativa $(\%)$ e as variáveis quantitativas pela média e desvio padrão. Foi utilizado o teste Qui-Quadrado para as associaçóes entre as variáveis categóricas, considerando nível de significância de $5 \%(\mathrm{p}<0,05)$, nos casos de constatação da associação global, realizou-se o cálculo dos resíduos ajustados. Para análise de correlação utilizou-se a correlaçáo de Spearman.

Os aspectos éticos da pesquisa com seres humanos do Conselho Nacional de Saúde foram respei- 
tados ${ }^{(17)}$ tendo aprovação do Comitê de Ética em Pesquisa sob no do parecer 2.237.779.

\section{Resultados}

Participaram da pesquisa 139 trabalhadores de enfermagem que atuavam no turno noturno durante o período de coleta de dados (41 enfermeiros, 76 técnicos em enfermagem, 22 auxiliares em enfermagem). A média de idade dos participantes foi de 42,6 anos (DP $\pm 9,47)$ (mínimo, 23; máximo, 69 anos) e a média do tempo de trabalho na unidade foi de 9,7 anos $(\mathrm{DP} \pm 8,12)$ (mínimo, 5; máximo, 38 anos).

Houve predominância dos trabalhadores do sexo feminino $86,3 \%(\mathrm{n}=120)$, com companheiro $82,7 \%(\mathrm{n}=115)$, filhos 79,9\% ( $\mathrm{n}=111)$ e pós-graduados 58,3\% (n=81). Relacionado à saúde, 29,5\% $(\mathrm{n}=41)$ realizavam tratamento de saúde, $40,3 \%$ $(\mathrm{n}=53)$ utilizavam medicação, 50,4\% $(\mathrm{n}=70)$ praticavam atividade física uma ou mais vezes na semana e $93,5 \%(n=130)$ utilizavam o tempo livre para lazer.

Referente às características laborais, 19,4\% $(\mathrm{n}=27)$ possuíam outro emprego, 70,5\% $(\mathrm{n}=98)$ receberam treinamento para trabalhar no setor, $95 \%(n=122)$ e $87,8 \%(n=129)$ estavam satisfeitos com trabalho e remuneração, respectivamente. Percentual de 35,3\% ( $n=49)$ sofreram acidentes e $18 \%(n=25)$ se afastaram do trabalho por motivo de doença nos últimos seis meses antecedentes à coleta. A maioria dos trabalhadores, 92,8\% ( $n=129)$, optou por trabalhar no turno noturno.

Ao avaliar os danos relacionados ao trabalho, identificou-se que a média de danos físicos foi 2,19 $(\mathrm{DP} \pm 1,18)$, o que o classificou em crítico. A média dos danos psicológicos foi de 1,11 $(\mathrm{DP} \pm 1,15)$ e dos danos sociais 1,27 (DP $\pm 1,10)$, classificando-os como suportáveis.

Visualiza-se (Tabela 1) o percentual de trabalhadores que apresentavam danos físicos, psicológicos e sociais relacionados ao trabalho, de acordo com a classificação.

Ao dicotomizar a classificação dos danos em adoecimento e não adoecimento identificou-se
Tabela 1. Classificação dos danos relacionados ao trabalho

\begin{tabular}{lccc}
\hline Classificação & $\begin{array}{c}\text { Danos físicos } \\
\mathbf{n}(\%)\end{array}$ & $\begin{array}{c}\text { Danos } \\
\text { psicológicos } \\
\mathbf{n}(\%)\end{array}$ & $\begin{array}{c}\text { Danos sociais } \\
\mathbf{n}(\%)\end{array}$ \\
\hline Suportável & $60(43,2)$ & $107(77,0)$ & $101(72,7)$ \\
Crítico & $45(32,4)$ & $18(12,9)$ & $32(23,0)$ \\
Grave & $26(18,7)$ & $11(7,9)$ & $4(2,9)$ \\
Doenças ocupacionais & $8(5,8)$ & $3(2,2)$ & $2(1,4)$ \\
\hline
\end{tabular}

$56,9 \%(n=79)$ em adoecimento físico, 23\% ( $n=32)$ em adoecimento psicológico e 27,3\% ( $n=38)$ em adoecimento social. Relacionado ao sono, houve predomínio da qualidade do sono ruim (80,6\%, $\mathrm{n}=112$ ). Apresenta-se (Tabela 2) a associação entre o adoecimento/não adoecimento físico, psicológico e social e a qualidade do sono.

Tabela 2. Associação entre adoecimento/não adoecimento físico, psicológico e social e qualidade do sono

\begin{tabular}{lccc}
\hline Variável & \multicolumn{2}{c}{ Qualidade do sono } & \\
& $\begin{array}{c}\text { Boa } \\
\mathrm{n}(\%)\end{array}$ & $\begin{array}{c}\text { Ruim } \\
\mathrm{n}(\%)\end{array}$ & p-value* \\
\hline Não adoecimento físico & $22(81,5)$ & $38(34)$ & $<0,001^{*}$ \\
\hline Adoecimento físico & $5(18,5)$ & $74(66)$ & \\
Não adoecimento psicológico & $24(88,9)$ & $83(74,1)$ & 0,101 \\
Adoecimento psicológico & $3(11,1)$ & $29(25,9)$ & \\
Não adoecimento social & $20(74,1)$ & $81(72,3)$ & 0,854 \\
\hline Adoecimento social & $7(25,9)$ & $31(27,7)$ & \\
\hline
\end{tabular}

*Teste Qui-Quadrado; *Associação significativa $p<0,05$

Evidenciou-se associação significativa entre adoecimento físico e qualidade do sono ruim $(p<0,001)$. Na associação entre as variáveis sociolaborais e a qualidade do sono, constatou-se significância entre qualidade do sono ruim e a variável sexo feminino $(\mathrm{p}=0,046)$. Ao associar as variáveis sociolaborais e o adoecimento/não adoecimento, encontrou-se associação significativa entre o adoecimento físico e as variáveis sexo feminino $(\mathrm{p}=0,001)$, não praticar atividade física $(\mathrm{p}=0,020)$, acidente de trabalho $(p=0,027)$ e afastamento do trabalho $(p=0,002)$. Identificou-se associação significativa entre adoecimento psicológico e as variáveis sexo feminino $(\mathrm{p}=0,005)$, tratamento de saúde $(\mathrm{p}=0,014)$, uso de medicação $(\mathrm{p}=0,004)$ e não praticar atividade física $(p=0,039)$. Observa-se na tabela 3 a correlação entre variáveis do estudo.

Identificou-se correlação direta entre idade e tempo de trabalho $(\mathrm{r}=0,634)$; danos físicos e psicológicos $(r=0,421)$; danos físicos e sociais $(r=0,322)$; 
Tabela 3. Matriz de correlação da idade, tempo de trabalho, danos físicos, psicológicos e sociais e qualidade do sono

\begin{tabular}{lccccc}
\hline Variável & Idade & $\begin{array}{c}\text { Tempo de } \\
\text { trabalho }\end{array}$ & $\begin{array}{c}\text { Danos } \\
\text { físicos }\end{array}$ & $\begin{array}{c}\text { Danos } \\
\text { psicológicos }\end{array}$ & $\begin{array}{c}\text { Danos } \\
\text { sociais }\end{array}$ \\
\hline Idade & & & & & \\
Tempo de trabalho & $0,634^{*}$ & & & & \\
Danos físicos & $-0,008$ & 0,049 & & & \\
Danos psicológicos & 0,159 & 0,161 & $0,421^{*}$ & & \\
Danos sociais & $-0,052$ & 0,012 & $0,322^{*}$ & $0,479^{*}$ & \\
PSQl global & $-0,076$ & 0,008 & $0,440^{*}$ & $0,238^{*}$ & 0,099 \\
\hline
\end{tabular}

*Coeficiente de Correlação de Spearman; * ${ }^{*}$ Correlação significante $p<0,01$

danos psicológicos e sociais ( $\mathrm{r}=0,479)$; PSQI global e danos físicos $(r=0,440)$ e entre PSQI global e danos psicológicos $(r=0,238)$.

\section{Discussão}

As limitações referem-se ao tipo de estudo transversal, que limita a possibilidade de estabelecer relaçóes causais. Considerando que a análise do estudo foi com os trabalhadores de enfermagem que atuavam no turno noturno, cabe aprofundar a investigação com esta população, por meio de pesquisas participativas, e, ainda, em futuras investigaçóes, incluir outros trabalhadores que atuam nesse turno com vista a comparar possíveis semelhanças ou divergências acerca dos fatores relacionados. Outra limitação refere-se ao viés de seleção, pois esse estudo desconsiderou trabalhadores em licença ou afastamento de qualquer natureza.

O intuito com essa pesquisa é de fornecer informaçóes sobre os fatores associados à qualidade do sono e adoecimento em trabalhadores de enfermagem que atuam no turno noturno para contribuir com o desenvolvimento de intervençóes, construídas juntamente com os gestores, maximizando a saúde do trabalhador com repercussóes positivas na assistência.

A partir da análise dos dados, identificou-se idade média de 42,6 anos, dado semelhante ao encontrado em estudo nacional realizado com essa população. ${ }^{(8)} \mathrm{A}$ idade foi correlacionada ao tempo de trabalho na unidade, ou seja, quanto maior a idade, maior o tempo de trabalho, o que sugere maior exposição do trabalhador ao contexto de trabalho. Sobre isso, estudo aponta que as dores no corpo e distúrbios biológicos relatados pelos profissionais de enfermagem, que podem ser decorrentes de postu- ras forçadas ou adotadas por um longo tempo, contribuem para o desenvolvimento de diversos problemas musculoesqueléticos, ${ }^{(13)}$ o que sinaliza para o risco de danos à saúde.

Predominaram as trabalhadoras com companheiros e filhos, dados similares a investigaçôes realizadas com trabalhadores de enfermagem. ${ }^{(18,19)}$ Houve associação do sexo feminino e adoecimento físico $(p=0,001)$, psicológico $(p=0,005)$ e qualidade do sono ruim $(p=0,046)$. A hegemonia do sexo feminino pode implicar em sobrecarga física e mental devido às atribuiçôes domésticas e familiares ainda prevalentes para estas. Essa sobrecarga pessoal pode influenciar nas atividades laborais, na qualidade da assistência prestada, tomada de decisóes e altos índices de distúrbios e estresse entre as trabalhadoras. ${ }^{(20)}$

Ao investigar os danos à saúde neste grupo de trabalhadores, identificou-se avaliação crítica para danos físicos, o que indica sofrimento no trabalho e sinaliza estado de alerta. O contexto de trabalho interfere na relação saúde-doença, causando o adoecimento físico e mental do trabalhador. ${ }^{(12)}$

Sobre a avaliação do sono, identificou-se predomínio da qualidade do sono ruim, dado convergente com estudo europeu que evidenciou que trabalhadores de enfermagem com atuação no noturno tinham sono de pior qualidade em relação aos que trabalhavam no turno diurno. ${ }^{(9)}$

Soma-se a isso a identificação de trabalhadores com qualidade do sono ruim associados ao adoecimento físico $(p<0,001)$. Isso sinaliza que a privação do sono afeta aspectos físicos e mentais, funções cognitivas e emocionais, podendo deprimir o sistema imunológico e provocar distúrbios metabólicos. ${ }^{(7)}$ Além disso, aumenta a secreção de cortisol, hormônio que contribui para distúrbios gastrointestinais, peso corporal, distúrbio de comportamento, hipertensão e osteoporose, ${ }^{(21)}$ situações que podem estar relacionadas ao comprometimento da saúde do trabalhador do turno noturno.

Esse dado é confirmado pela correlação entre qualidade do sono e danos físicos e danos psicológicos, sugerindo que quanto pior a qualidade do sono, mais dores no corpo, distúrbios biológicos e mau humor. Pesquisa realizada na Alemanha com trabalhadoras de enfermagem identificou que alteraçóes 
físicas como taquicardia, problemas circulatórios e dores nas costas constantes estavam correlacionados a uma pior qualidade do sono. ${ }^{(10)}$ Além disso, o sono comprometido na sua quantidade/qualidade favorece a fadiga, definida pela sensação de desgaste, cansaço e falta de energia, ${ }^{(6)}$ o que pode contribuir para a ocorrência de danos à saúde.

$\mathrm{O}$ trabalho de enfermagem requer alto grau de atenção, concentração, destreza e responsabilidade que, somado ao turno noturno, às longas jornadas e à qualidade do sono ruim, pode repercutir em prejuízo na saúde. A análise dos dados mostrou associação entre adoecimento psicológico, tratamento de saúde $(\mathrm{p}=0,014)$ e uso de medicação $(\mathrm{p}=0,004)$. Pesquisa realizada com 502 trabalhadores de enfermagem de uma instituição hospitalar que investigou os fatores associados à síndrome de Burnout, segundo o turno de trabalho, evidenciou que aqueles que atuavam no turno noturno estavam insatisfeitos com o sono e apresentaram maiores chances de alta exaustão emocional. ${ }^{(22)}$

A exaustão pode favorecer o adoecimento físico, que apresentou diferença significativa com acidente de trabalho $(\mathrm{p}=0,027)$ e afastamento do trabalho $(\mathrm{p}=0,002)$, ou seja, foram identificadas pessoas com dores no corpo e distúrbios biológicos associadas ao acidente de trabalho e/ou afastamento por motivo de doença. A saúde do trabalhador que atua no turno noturno precisa ser (re) planejada por meio de açôes institucionais que o sensibilizem para o cuidado de si.

Dentre as açôes, cita-se o estímulo à prática de atividade física, que nesse estudo foi associada ao não adoecimento físico $(\mathrm{p}=0,020)$ e não adoecimento psicológico $(p=0,039)$, o que reforça o entendimento sobre os benefícios desta prática para a saúde. Dados de pesquisa ${ }^{(23)}$ realizada com 235 trabalhadores de enfermagem e médicos atuantes em diferentes turnos identificou a prática de exercício físico diário como fator de proteção, com melhorias no condicionamento físico, nas relaçôes familiares e sociais e na minimização dos sentimentos negativos.

Assim, partir do exposto, constata-se a necessidade de açóes para melhorias nos contextos de trabalho e promoçáo da saúde do trabalhador de enfermagem, ${ }^{(24)}$ devendo considerar as particularidades relacionadas ao turno noturno. Por fim, ao refletir sobre o processo de trabalho de enfermagem e a saúde do trabalhador, é imprescindível atentar para a avaliação dos danos relacionados ao trabalho, especialmente os físicos, e da qualidade do sono, visto que obtiveram os resultados mais negativos no grupo de trabalhadores investigados.

\section{Conclusão}

Essa pesquisa identificou predomínio da qualidade do sono ruim em trabalhadores de enfermagem que atuam no turno noturno, adoecidos fisicamente. Os fatores associados à pior qualidade do sono foram sexo e danos físicos; associados ao adoecimento físico, as variáveis sexo, prática de atividade física, acidente de trabalho e afastamento do trabalho; e associados ao adoecimento psicológico as variáveis sexo, tratamento de saúde, uso de medicação e prática de atividade física. Ofertar espaços para que os trabalhadores possam se informar e discutir sobre os fatores que podem comprometer a sua saúde, com o objetivo de instrumentaliza-los para o cuidado de si, poderá repercutir na assistência.

\section{Agradecimentos}

Ao Conselho Nacional de Desenvolvimento Científico e Tecnológico (CNPq) pelo financiamento por meio do Edital Universal 2016, processo no 402986/2016-4 e à Coordenação de Aperfeiçoamento de Pessoal de Nível Superior (CAPES) pela bolsa de mestrado.

\section{Colaborações}

Cattani AN, da Silva RM, Beck CLC, Miranda FMA, Dalmolin GL e Camponogara S contribuíram com a concepção do estudo, análise e interpretação dos dados, elaboração do artigo, revisão critica do conteúdo e aprovação final da versão a ser publicada. 


\section{Referências}

1. Mauro MY, Rebelo AM, Ferreira AO, Sper NP, Santos MI, Gallasch CH. Trabalho noturno e alterações de peso corporal autopercebidas pelos profissionais de enfermagem. Rev Enferm UERJ. 2019;27:e31273.

2. Vetter C, Devore EE, Wegrzyn LR, Massa J, Speizer FE, Kawachi I, et al. Association between rotating night shift work and risk of coronary heart disease among women. JAMA. 2016;315(16):1726-34.

3. Simões J, Bianchi LR. Prevalence of the burnout syndrome and sleep quality of nursing technical workers. Saúde Pesqui. 2016;9(3):473-81.

4. Marçal JA, Moraes BF, Mendes SS, De Martino MM, Sonati JG. Sono e variáveis de saúde de profissionais de enfermagem nos diferentes turnos de trabalho. REME Rev Min Enferm. 2019;23:e-1235.

5. Nascimento JO, Santos JD, Meira KC, Pierin AM, Souza-Talarico JN. Shift work of nursing professionals and blood pressure, burnout and common mental disorders. Rev Esc Enferm USP. 2019;53:e03443.

6. Fernandes BK, Ribeiro AC, Borges CL, de Galiza FT, Joventino ES. Influences of night shifts in sleeping of nursing workers: integrative review. Rev Enferm Atual. 2017;81(19):97-103.

7. Bonanno L, Metro D, Papa M, Finzi G, Maviglia A, Sottile F, et al. Assessment of sleep and obesity in adults and children: observational study. Medicine (Baltimore). 2019;98(46):e17642.

8. Viana MC, Silva KK, De Martino MM, Bezerra CM, Oliveira AP, Souza AM, et al. Qualidade de vida e sono de enfermeiros nos turnos hospitalares. Rev Cubana Enferm. 2019;35(2):1-14

9. Gómez-García T, Ruzafa-Martínez M, Fuentelsaz-Gallego C, Madrid JA, Rol MA, Martínez-Madrid MJ, et al.; SYCE and RETICEF Group. Nurses' sleep quality, work environment and quality of care in the Spanish National Health System: observational study among different shifts. BMJ Open. 2016;6(8):e012073.

10. Hasan AA, Tumah $H$. The correlation between occupational stress coping strategies, and the levels of psychological distress among nurses working in mental health hospital in Jordan. Perspect Psychiatr Care. 2019;55(2):153-60.

11. Mendes AM, Ferreira MC. Inventário sobre o trabalho e riscos de adoecimento - ITRA: Instrumento auxiliar de diagnóstico de indicadores críticos no trabalho. In: Mendes AM, editor. Psicodinâmica do trabalho: teoria, método e pesquisas. São Paulo: Casa do Psicólogo; 2007.
12. Guimarães AL, Felli VE. Notification of health problems among nursing workers in university hospitals. Rev Bras Enferm. 2016;69(3):507-14.

13. Prestes FC, Beck CL, Magnago TS, Silva RM, Coelho AP. Health problems among nursing workers in a haemodialysis service. Rev Gaucha Enferm. 2016;37(1):e50759.

14. Brasil. Ministério da Educação. Empresa Brasileira de Serviços Hospitalares (EBSERH). Norma Operacional DGP nº 04/2017 - Escalas de trabalho. Brasilia (DF): Ministério da Educação; 2017.

15. Bertolazi AN, Fagondes SC, Hoff LS, Dartora EG, Miozzo IC, de Barba $\mathrm{ME}$, et al. Validation of the Brazilian Portuguese version of the Pittsburgh Sleep Quality Index. Sleep Med. 2011;12(1):70-5.

16. Pinto J, Perin C, Dick NR, Lazzarotto AR. Avaliação do Sono em um Grupo de Policiais Militares de Elite. Acta Paul Enferm. 2018;31(2):153-61.

17. Brasil. Ministério da Saúde. Conselho Nacional de Saúde. Resolução № 466, de 12 de dezembro de 2012. Diretrizes e normas regulamentadoras sobre pesquisa envolvendo seres humanos. Brasília (DF): CNS; 2012.

18. Silva RM, Beck CL, Prestes FC, Cigana FA, Trindade ML, Santos IG. Excessive daytime sleepiness and health damage in nursing clinic surgical workers. Texto Contexto Enferm. 2019;28:e20170455.

19. Worm FA, Oliveira Pinto MA, Schiavenato D, Amora Ascari R, De Lima Trindade L, Martins da Silva O. Risco de adoecimento dos profissionais de enfermagem no trabalho em atendimento móvel de urgência. Rev Cuid (Bucaramanga). 2016;7(2):1288-96.

20. de Araujo MA, Lunardi Filho WD, Alvarenga MRM, de Oliveira RD, Souza JC, Vidmantas S. Perfil sociodemográfico dos enfermeiros da rede hospitalar. Rev Enferm UFPE online. 2017; 11(Supl.11):4716-25.

21. Silva RM, Goulart CT, Guido LA. Evolução histórica do conceito de estresse. REVISA. 2018;7(2):148-56.

22. Vidotti V, Ribeiro RP, Galdino MJ, Martins JT. Burnout Syndrome and shift work among the nursing staff. Rev Lat Am Enfermagem. 2018;26:e3022.

23. Portero de la Cruz S, Vaquero Abellán M. Professional burnout, stress and job satisfaction of nursing staff at a university hospital. Rev Lat Am Enfermagem. 2015;23(3):543-52.

24. Oliveira AL, da Costa GR, Fernandes MA, Gouveia MT, Rocha SS. Presenteísmo, fatores de risco e repercussões na saúde do trabalhador de enfermagem. Av Enferm. 2018;36(1):79-87. 